\title{
Trends and Out Look for Clinical Diagnostic Testing
}

\author{
Raghavendra Rao M.V ${ }^{1}$., Sireesha Bala ${ }^{1}$, Sripada Pallavi. ${ }^{2}$., \\ KrishnaSowmya. $\mathbf{M}^{3}$.,MahendraKumar ${ }^{4}$, Resma Fathe, ${ }^{1}$ SamirFatteh and Sateesh.Babu. ${ }^{1}$ \\ ${ }^{1}$ Avalon University School of Medicine, Curacao, Central America. \\ ${ }^{2}$ Apollo Institute of Medical Science and Research Institute, Jubilee Hills, Hyderabad, Telangana, India, \\ ${ }^{3}$ Burjil Hospital, Abu Dhabhi, United Arab Emirates \\ ${ }^{4}$ Indian Institute of Science Education and Research, Bhopal \\ reachdrmvrrao@gmail.com; dr.raghavendra@avalonu.org
}

\section{PREPHASE}

There are several approaches are being used in clinical diagnosis including routine laboratory tests as conventional methods and also rapid and robust modern methods. Both the approaches have their significance in the clinical diagnosis of a disease. The conventional approaches largely depend on microscopy and staining of microorganism responsible for disease while modern methods are based on molecular signature and findings. In the current scenario, there is a need for rapid and robust diagnostics as global disease burden is prevailing. At the same time, there is the continuous emergence of new and complex infectious pathogens, and hence early diagnosis provides ease in disease management. Recently, use of nanotechnology and enzyme-based diagnostic became popular and shown satisfactory results. The use of diagnostic become an integral part of modern medicine and involves molecular biology and cutting-edge bio-engineering as well. The integration of information technology in diagnostic is an added advantage to minimize time and ensure precise data interpretation.

Keywords: Polymerase chain reaction (PCR), Nucleic acid-based tests, A rapid diagnostic test (RDT), Readymade diagnostic kits, Patient compliance, genomics

\section{Introduction}

Medical diagnosis is the condition explains a person's symptoms and signs (1). It is most often referred to as diagnosis with the medical context being implicit. There are several means of diagnosis of diseases including symptomatic, molecular and genomics (2). The conventional way of clinical diagnosis is still in practice provide first and basic information regarding disease and causative agents as well. (3) Integration is influenced by the clinical syndrome, the availability of and access to appropriate diagnostics, the place of service, and the experience and knowledge of the healthcare provider (4). The goal of the chapter is to increase awareness of the current and potential value of infectious diseases diagnostics for patient care and public health and to promote further development of needed diagnostics. 
The credit goes to Antonie van Leeuwenhoek (1632-1723), the "father of the microscope." (5). . The field of clinical microbiology is currently in transition, and standard-of-care testing is now a hybrid of old and new methodologies (6). The evolution of infectious diseases diagnostics has resulted from advances in chemistry, immunology, molecular biology, engineering, automation, and nucleic acid amplification. (7). Individual pathogens can be readily identified in a wide variety of specimen types including blood, urine, tissue, mucosal swabs, cerebrospinal fluid (CSF), respiratory secretions, and stool samples. The modern diagnostic is quick, robust and precise in finding cause for candidate disease and allow ease in data studies (8).

One basic and very old method is the identification of a microorganism simply by looking at it under a microscope. Most samples are treated for stains. Stains are special dyes that color the microorganisms, causing them to stand out from the background (11). Doctors add substances to the dish or testtube to stop the growth of microorganisms that donot cause the disease . (12). Many microorganisms, such as the bacteria that cause urinary tract infections or strep throat, can easily be grown in culture. Some bacteria, such as the bacteria that cause syphilis, cannot be cultured at all (13). They are produced by certain types of white blood cell when these white blood cells encounter a foreign substance or cell (14).If a person has antibodies to a particular microorganism, it means that the person has been exposed to that microorganism and has mounted an immune response (15). Antigen tests detect the presence of a microorganism directly so that doctors can diagnose an infection quickly, without waiting for a person to produce antibodies in response to the microorganism (16). If there are antigens from that microorganism in the person's sample, they attach to the test antibody (17). D This genetic material consists of nucleic acids: deoxyribonucleic acid (DNA) or ribonucleic acid (RNA). The polymerase chain reaction (PCR) is an example of this type of test (18).

Thus, these tests are done only when a doctor already suspects a particular disease. Most nucleic acidbased tests are designed to identify the presence of a microorganism (called qualitative testing) (19). However, a few of these tests can measure the amount of genetic material present (called quantitative testing) in certain microorganisms, such as HIV and hepatitis $C$, and thus determine how severe the infection is. Quantitative tests can also be used to monitor how well treatment is working. Nucleic acidbased tests can sometimes be used to check the microorganisms for genes or gene mutations that make the microorganism resistant to a drug (20) Enzymes produced by the microorganism (which help the microorganism infect cells or spread through tissues faster) (21). For many diseases, the clinical laboratory provides essential diagnostic information (22).A rapid diagnostic test (RDT) is a medical diagnostic test that is quick and easy to perform. RDTs are suitable for preliminary or emergency medical screening and use in medical facilities with limited resources (23). Malaria RDTs detect specific antigens (proteins) produced by malaria parasites that are present in the blood of infected individuals. Some RDTs detect a single species (either P. falciparum or P. vivax), some detect multiple species ( $P$. falciparum, P. vivax, P. malariae and P. ovale) and some further distinguish between P. falciparum and non-P. Falciparum infection, or between specific species. Blood for the test is commonly obtained from a finger-prick and results are available within 15-30 minutes (24)With the use of conventional diagnostic methods and new cutting-edge modern tools in clinical diagnosis outcomes are- Rapid clinical diagnosis Robust tests and higher reproducibility ,Readymade diagnostic kits ,Ready to use, Ease in sample 
Raghavendra Rao M.V., Sireesha Bala, Sripada Pallavi.T., KrishnaSowmya. M.,MahendraKumar.,Resma Fathe, SamirFatteh and Sateesh.Babu.A; Trends and Out Look for Clinical Diagnostic Testing.. Journal of Biomedical Engineering and Medical Imaging, Volume 5, No 4, August (2018), pp 1-8

processing ,Digital output, Ease of data interpretation ,Low title volume Patient compliance and Low cost

\section{History and Mechanism}

Patient safety agenda is gaining momentum in the health care systems of all developed countries. However, adverse event detection systems and initiatives to reduce error rates in medicine are in their infancy. Laboratory services play a crucial role in both individual and population-based healthcare, and clinical laboratories use many different methods to reduce errors, ensure patient safety, and improve quality including quality control procedures, quality assurance programs, accreditation of laboratories and certification of education programs. Considerable advances in analytical techniques, laboratory instrumentation, information technologies, automation and organization have granted an exceptional degree of analytical quality over the past 50 years. This, in turn, has resulted in a significant decrease in error rates, analytical error rates in particular. There is consolidated evidence that nowadays, most laboratory errors fall outside the analytical phase, and that pre- and post-analytical processes are more vulnerable to error than analytical processes. The first recorded examples of medical diagnosis are found in the writings of Imhotep (2630-2611 $\quad$ BC) in ancient Egypt (the Edwin Smith (25) Babylonianmedical textbook, the Diagnostic Handbook written by Esagil-kin-apli (fl.10691046 BC), introduced the use of empiricism, logic and rationality in the diagnosis of an illness or disease (26) Traditional Chinese Medicine, as described in the Yellow Emperor's Inner Canon or Huangdi Neijing, specified four diagnostic methods: inspection, auscultation-olfaction, interrogation, and palpation.(27)Hippocrates was known to make diagnoses by tasting his patients' urine and smelling their sweat (28) The credit goes to Antonie van Leeuwenhoek (1632-1723), the "father of the microscope," changed the course of infectious diseases when he enabled the visualization of the microbial world, a world no one had imagined. Medical diagnosis is the process of determining which disease or condition explains a person's symptoms and signs (29). It is most often referred to as diagnosis with the medical context being implicit. The diagnosis is the first step in the process of therapeutics prescription. There are several means of diagnosis of diseases including symptomatic, molecular and genomics (30). Diagnostic tests play a major role in the clinical care of patients with infectious diseases, including detection of specific pathogens, the discovery of new pathogens, determining appropriate therapy, monitoring response to therapy, assessing prognosis, and disease surveillance (31). Integration is influenced by the clinical syndrome, the availability of and access to appropriate diagnostics, the place of service, and the experience and knowledge of the healthcare provider (32). The goal of the chapter is to increase awareness of the current and potential value of infectious diseases diagnostics for patient care and public health and to promote further development of needed diagnostics.

\section{Major Advances and Discoveries}

Advanced clinical diagnostics has fully equipped with ware housing, animal facilities, clean room, high speed and ultra centrifuges, iso environmental chambers for various atmospheric conditions of temp and air mixtures, fer mentors with high speed filtration and separation, laminar flow biosafty and equipped for biological, microbiology, tissue culture, large scale cryopreservation, biochemical, analytical, virology, immunology, food and safety, cryopreservation of biological materials and other investigative work The old and new diagnostic tests to diagnose pathogenic parasites, viruses,bacteria 
are observing parasites,ova,cysts, blood smear,MRI,CAT,ELISA (Enzyme linked immunosorbent assay),H A(Hem agglutination),CF (Complement fixation) FAST(Falcon assay screening test),DOT-ELISA,RDTS (Rapid antigen detecting system),LIPS(Luciferase Immunoprecipitation system, PCR(Polymerase chain reaction),RT-PCR (Real time Polymerase chain reaction),LAMP (Loop mediated isothermal Amplification) etc are the major diagnostic tests.

\section{Ideas Where the Research Go Next}

The clinical diagnosis is a first and most crucial step in the process of finding therapeutics for a disease. There are several approaches are being used in clinical diagnosis including routine laboratory tests as conventional methods and also rapid and robust modern methods. Both the approaches have their significance in the clinical diagnosis of a disease. The conventional approaches largely depend on microscopy and staining of microorganism responsible for disease while modern methods are based on molecular signature and findings. In 19 the current scenario, there is a need for rapid and robust diagnostics as global disease burden is prevailing. At the same time, there is the continuous emergence of new and complex infectious pathogens, and hence early diagnosis provides ease in disease management. Recently, use of nanotechnology and enzyme-based diagnostic became popular and shown satisfactory results. The use of diagnostic become an integral part of modern medicine and involves molecular biology and cutting-edge bio-engineering as well. The integration of information technology in diagnostic is an added advantage to minimize time and ensure precise data interpretation.

A medical error is a preventable adverse effect of care, whether or not it is evident or harmful to the patient.Laboratory blood studies can reveal a little information about organ systems throughout the body. The amount of blood taken for a laboratory test is not harmful. Human body manufactures a couple of milliliters of new blood every day. Blood studies may give information about the levels of sodium, potassium, calcium and other chemicals. Presence of certain enzymes and information about the coagulation characteristics, levels of sugar, urea, cholesterol, alcohol, protein, and other drugs of patients blood sample.

Diagnostic error can be defined as a diagnosis that is missed, wrong or delayed, as detected by some subsequent definitive test or finding. The ensuing harm results from the delay or failure to treat a condition present when the working diagnosis was wrong or unknown, or from treatment provided for a condition not actually present.

\section{Significant Gap in Research}

Mistakes and failures are integral part of any great effort worth the mention.Your best teacher is your last mistake. Developing new manifolds by revitalizing old ideas. The quality of thinking action decide your strength. Is it a sin or boon to admit patient in a hospital for treatment? . Medical error is not included on death certificates or in rankings of cause of death. Hospital is expected to be a safe place. When you take a sick patient to the hospital, you expect that the patient is in good hands and under the umbrella of experienced doctors. Errors in diagnosis, misdiagnosis of psychological disorder, competency, education and training are the main features causing fatal deaths. These are the common misconceptions about adverse events, and the arguments and explanations against those misconceptions are noted in parentheses: "Bad apples" or incompetent health care providers are a common cause. (Although human error is commonly an initiating event, the faulty process of delivering 
Raghavendra Rao M.V., Sireesha Bala, Sripada Pallavi.T., KrishnaSowmya. M.,MahendraKumar.,Resma Fathe, SamirFatteh and Sateesh.Babu.A; Trends and Out Look for Clinical Diagnostic Testing.. Journal of Biomedical Engineering and Medical Imaging, Volume 5, No 4, August (2018), pp 1-8

care invariably permits or compounds the harm, and is the focus of improvement.(33) High risk procedures or medical specialties are responsible for most avoidable adverse events. (Although some mistakes, such as in surgery, are harder to conceal, errors occur in all levels of care. Even though complex procedures entail more risk, adverse outcomes are not usually due to error, but to the severity of the condition being treated(34) However, USP has reported that medication errors during the course of a surgical procedure are three times more likely to cause harm to a patient than those occurring in other types of hospital care(35) If a patient experiences an adverse event during the process of care, an error has occurred. (Most medical care entails some level of risk, and there can be complications or side effects, even unforeseen ones, from the underlying condition or from the treatment itself) (36)

\section{Current Debate}

Incorrect laboratory tests account for significant harm. Researchers estimated that the number of patients suffering from missed diagnostic tests are annually in thousands. These are potentially preventable, subject to the condition if proper attention is paid.

Diagnosing diseases and disorders requires highly developed skill on the part of the physician or other medical professional .Usually the diagnosis calls for systematic use of instruments and diagnostic aids, various tests, and , often with sophisticated instruments and machines.

Quality systems are the mainstay of clinical laboratory management. The comprehensive laboratory testing process must be continually monitored and evaluated to ensure reliable test results and set the foundation for quality improvement. While such efforts have resulted in significant improvements in many of the processes, errors still occur. In order to implement corrections and improve the testing process, the laboratorian must identify the various sources of errors.

Last two decades has witnessed phenomenal advances in the field of medicine following revolutionary changes taking place in the application of technology and as a consequence, Biochemistry and Microbiology have evolved as the most important branch of evidence based medicine.

The quality of any laboratory test result is dependent on many variables, It begins with skill, and knowledge when preparing the patient and specimen are essential to the provision of the highest quality standards for testing and services. The patient must first be properly prepared so that the best possible specimen can be collected. Next, the actual collection of the specimen must be completed. Then, the specimen should be properly processed, packaged and transported to the laboratory in a timely manner and under environmental conditions that will not compromise the integrity of the specimen. After all of these activities take place, a quality analysis can be performed.

Some common reasons of not getting quality and reliable results are 1. Lack of commitment on part of staff performing the tests 2. Poor management and supervision 3. Poor understanding of quality assurance concepts 4 . Analysts do not understand the concepts of assay principles 5 . Reagents used are not high quality 6 . Poor quality of instruments 7. Procedures are not followed as recommended 8 . Under staff leads to high error rate. 9. Lack of equipment. 10. Mislabeling blood sample. 11. Un labeling blood sample. 


\section{REFERENCES}

[1] Ross AGP, Bartley PB, Sleigh AC, Olds GR, Li Y, Williams GM, McManus DP. Schistosomiasis. N Eng J Med 2002;346:1212-9.

[2] Gryseels B, Polman K, Clerinx J, Kestens L. Human schistosomiasis. Lancet 2006;368:1106-18.

[3] Steinmann P, Keiser J, Bos R, Tanner M, Utzinger J. Schistosomiasis and water resources development: systematic review, meta-analysis, and estimates of people at risk. Lancet Infect Dis 2006;6:411-25.

[4] King CH, Dickman K, Tisch DJ. Reassessment of the cost of chronic helminthic infection: a meta-analysis of disability-related outcomes in endemic schistosomiasis. Lancet 2005;365:561-9.

[5] King CH, Dangerfield-Cha M. The unacknowledged impact of chronic schistosomiasis. Chronic Illness 2008;4:65-79.

[6] Mas-Coma S, Valero MA, Bargues MD. Climate change effects on trematodiases, with emphasis on zoonotic fascioliasis and schistosomiasis. Vet Parasitol 2009;163:264-80

[7] Leshem E, Meltzer E, Marva E, Schwartz E. Travel-related schistosomiasis acquired in Laos. Emerg Infect Dis 2009;15:1823-6.

[8] Ross AG, Vickers D, Olds GR, Shah SM, McManus DP. Katayama syndrome. Lancet Infect Dis 2007;7:21824.

[9] Wynn TA, Thompson RW, Cheever AW, Mentink-Kane MM. Immunopathogenesis of schistosomiasis. Immunol Rev 2004;201:156-67.

[10] Burke ML, Jones MK, Gobert GN, Li YS, Ellis MK, McManus DP. Immunopathogenesis of human schistosomiasis. Parasite Immunol 2009;31:163-76.

[11] Carod-Artal FJ. Neurological complications of Schistosoma infection. Trans R Soc Trop Med Hyg 2008;102:107-16.

[12] Ferrari TC. Involvement of the central nervous system in the schistosomiasis. Mem Inst Oswaldo Cruz 2004;99:59-62.

[13] McManus DP, Gray DJ, Li YS, Feng Z, Williams GM, Stewart D, et al. Schistosomiasis in the Peoples' Republic of China: the era of the Three Gorges Dam. Clinic Microbiol Rev 2010;23:442-66.

[14] Katz N, Chaves A, Pellegrino J. A simple device for quantitative stool thick-smear technique for schistosomiasis mansoni. Rev Inst Med Trop Sao Paulo 1972;14:397-400.

[15] Oliveira LM, Santos HL, Gonçalves MM, Barreto MG, Peralta JM. Evaluation of polymerase chain reaction as an additional tool for the diagnosis of low-intensity Schistosoma mansoni infection. Diagn Microbiol Infect Dis 2010;68:416-21.

[16] Wichmann D, Panning M, Quack T, Kramme S, Burchard GD, Grevelding C, Drosten C. Diagnosing schistosomiasis by detection of cell-free parasite DNA in human plasma. PLoS Negl Trop Dis 2009;3:e422. 
Raghavendra Rao M.V., Sireesha Bala, Sripada Pallavi.T., KrishnaSowmya. M.,MahendraKumar.,Resma Fathe, SamirFatteh and Sateesh.Babu.A; Trends and Out Look for Clinical Diagnostic Testing.. Journal of Biomedical Engineering and Medical Imaging, Volume 5, No 4, August (2018), pp 1-8

[17] Gomes LI, Dos Santos Marques LH, Enk MJ, de Oliveira MC, Coelho PM, Rabello A. Development and evaluation of a sensitive PCR-ELISA system for detection of Schistosoma infection in feces. PLoS Negl Trop Dis 2010;4:e664.

[18] Ellis MK, Li Y, Hou X, Chen H, McManus DP. sTNFR-II and sICAM-1 are associated with acute disease and hepatic inflammation in schistosomiasis japonica. Int J Parasitol 2008;38:717-23.

[19] Bergquist R, Johansen MV, Utzinger J. Diagnostic dilemmas in helminthology: what tools to use and when? Trends Parasitol 2009;25:151-6.

[20] Chand MA, Chiodini PL, Doenhoff MJ. Development of a new assay for the diagnosis of schistosomiasis, using cercarial antigens. Trans R Soc Trop Med Hyg 2010;104:255-8.

[21] Stothard JR, Sousa-Figueiredo JC, Standley C, Van Dam GJ, Knopp S, Utzinger J, et al. An evaluation of urine-CCA strip test and fingerprick blood SEA-ELISA for detection of urinary schistosomiasis in schoolchildren in Zanzibar. Acta Trop 2009;111:64-70.

[22] Doenhoff MJ, Cioli D, Utzinger J. Praziquantel: mechanisms of action, resistance and new derivatives for schistosomiasis. Curr Opin Infect Dis 2008;21:659-67. [PubMed]

[23] Cioli D, Pica-Mattoccia L. Praziquantel. Parasitol Res 2003;90:S3-S9. [PubMed]

[24] Hou XY, McManus DP, Gray DJ, Balen J, Luo XS, He YK, et al. A randomized, double-blind, placebocontrolled trial of safety and efficacy of combined praziquantel and artemether treatment for acute

[25] "Edwin Smith Papyrus". Retrieved 2015-02-28.

[26] H. F. J. Horstmanshoff, Marten Stol, Cornelis Tilburg (2004), Magic and Rationality in Ancient Near Eastern and Graeco-Roman Medicine, p. 97-98, Brill Publishers, ISBN 90-04-13666-5.

[27] Jingfeng, C. (2008). "Medicine in China". Encyclopaedia of the History of Science, Technology, and Medicine in Non-Western Cultures. pp. 1529-1534. doi:10.1007/978-1-4020-4425-0_8500. ISBN 978-14020-4559-2.

[28] "What Would Hipocrates Do?". Retrieved February 28, 2015.

[29] Ross AGP, Bartley PB, Sleigh AC, Olds GR, Li Y, Williams GM, McManus DP. Schistosomiasis. N Eng J Med 2002;346:1212-9.

[30] Gryseels B, Polman K, Clerinx J, Kestens L. Human schistosomiasis. Lancet 2006;368:1106-18.

[31] Steinmann P, Keiser J, Bos R, Tanner M, Utzinger J. Schistosomiasis and water resources development: systematic review, meta-analysis, and estimates of people at risk. Lancet Infect Dis 2006;6:411-25.

[32] King CH, Dickman K, Tisch DJ. Reassessment of the cost of chronic helminthic infection: a meta-analysis of disability-related outcomes in endemic schistosomiasis. Lancet 2005;365:561-9. 
Journal of Biomedical Engineering and Medical Imaging, Volume 5, No 4, Aug 2018

[33] Weingart SN, Wilson RM, Gibberd RW, Harrison B; Wilson; Gibberd; Harrison (March 2000). "Epidemiology of medical error". BMJ. 320 (7237): 774-

7. doi: $\underline{10.1136 / \mathrm{bmj} .320 .7237 .774}$. PMC $\underline{1117772}$. PMID $\underline{10720365}$

[34] Gardner, Amanda (6 March 2007). "Medication Errors During Surgeries Particularly Dangerous". The Washington Post. Retrieved 2007-03-13.

[35] US Agency for Healthcare Research \& Quality (2008-01-09). "Physicians Want To Learn from Medical Mistakes but Say Current Error-reporting Systems Are Inadequate". Retrieved 2008-03-23.

[36] Institute of Medicine (2000). To Err Is Human: Building a Safer Health System. Washington, DC: The National Academies Press. doi: $\underline{10.17226 / 9728}$. ISBN $\underline{\text { 978-0-309-26174-6 }}$ 